\title{
Entwicklung von Enzymelektroden zur amperometrischen Bestimmung von Lactatkonzentrationen
}

\author{
Christian Schubert $^{a}$, Sinan Ünlübayir ${ }^{b^{*}}$, Andreas Goehlich $^{c}$, \\ Hoc Khiem Trieu ${ }^{b}$ \\ Fraunhofer-Institut für Mikroelektronische Schaltungen und Systeme
}

Aktuelle Kontakte:
a: Tel.-Nr.: +49 (0)941 201-5802, E-Mail: christian.schubert@infineon.com
b. Technische Universität Hamburg-Harburg Institut für Mikrosystemtechnik Eißendorfer Straße 42, 21073 Hamburg
Tel.-Nr.: +49 (0)40 42878-2321, E-Mail: sinan.uenluebayir@tuhh.de
c: Fraunhofer-Institut für Mikroelektronische Schaltungen und Systeme
Finkenstrasse 61, 47057 Duisburg
Tel.-Nr.: +49 (0)203 3783 -129, E-Mail: andreas.goehlich@ims.fraunhofer.de

Die steigende Nachfrage nach portablen Geräten zur Kontrolle von medizinischen oder physikalischen Parametern für die Überwachung des Gesundheitszustands führt zu mehreren Ansätzen für Handheld oder Point-of-Care-Geräten. Diese finden Anwendung in der Medizin, in der Heimpflege oder auch im Lifestyle-Bereich. Die Patienten oder ganz allgemein die Benutzer werden ein solches Messgerät weniger in der Hand, sondern eher an der Kleidung oder am Körper befestigt tragen. Demnach müssen diese Messgeräte in Bezug auf Größe und Gewicht weiter verkleinert werden. Insbesondere elektrochemische Sensoren sind potentiell gut geeignet um diese Größenanforderungen zu erfüllen. In der vorliegenden Arbeit liegt das Augenmerk auf der Detektion von Laktat. Die Technik zur Herstellung der Enzymelektroden lässt sich jedoch auch auf andere elektrochemische Sensoren übertragen. Lactat ist das Salz der Milchsäure und entsteht als Stoffwechselprodukt bei der Energiebereitstellung im Körper durch unvollständige Verbrennung von Kohlenhydraten. Bedeutung hat es insbesondere für die Sportmedizin zur Beurteilung und Steuerung der Ausdauerleistungsfähigkeit. In der Notfall- und Intensivmedizin dient Lactat als ein potentielles Alarmsignal bei allgemeinen gesundheitlichen Problemen. Ein allgemeines Ziel ist es Lactat-Sensoren für in-vivo-Messungen einzusetzen. Beispielsweise sollen Sportler die Möglichkeit bekommen ihre Laktatkonzentration kontinuierlich über die Zeit einer Trainingseinheit zu messen, die Ergebnisse der Messungen dienen dann der Optimierung des Trainings. Dies würde eine Verbesserung gegenüber den etablierten Verfahren, welche nur die Messung von Einzelwerten vor und nach einer Trainingseinheit gestatten, bedeuten. Hierzu müssen Mikroelektroden mit entsprechend kleinen aktiven Sensoroberflächen entwickelt werden, um z.B. eine Integration in ein Hohl-Nadel- bzw. Nadel-Array-System zu ermöglichen. Hier wird von der Herstellung von Au-Elektroden mit Oberflächen im Mikrometerbereich berichtet, welche für den in-vitro-Einsatz in einem amperometrischen Lactat-Sensor mit einem Enzym funktionalisiert wurden. Als Enzym wurde Lactatoxidase verwendet und in einer Matrix aus Glutaraldehyd auf der Oberfläche der Elektroden immobilisiert.

Lactatoxidase ist ein Enzym, das eine chemische Reaktion katalysiert, in der Lactat-Säure mit Sauerstoff zu Pyruvat und Wasserstoffperoxid reagiert. Der amperometrisch messbare Redoxstrom, der bei der elektrokatalysierten Reaktion an Wasserstoffperoxid durch Elektronenübertragung entsteht, ist proportional zu der Konzentration von Lactat-Säure ${ }^{1}$.

Es wird eine Immobilisierungsmethode vorgestellt, welche sowohl die Aktivität des Enzyms bewahrt als auch eine ausreichende Haftung gewährleistet. Es wurden in Acryl eingebettete Au-Drahtelektroden mit Durchmessern von $200 \mu \mathrm{m}$ und $25 \mu \mathrm{m}$ sowie Dünnschicht Au-Elektroden mit einer Fläche von $100 \mathrm{~mm}^{2}$ hergestellt und vergleichend untersucht. Des Weiteren wurde der Einfluss einer Polymer-Membran (Nafion ${ }^{\circledR}$ ) auf die Funktionalität der Elektroden untersucht. Zur Charakterisierung der Elektroden wurden cyclovoltametrische Messungen (CV) durchgeführt. 


\section{Herstellung der Elektroden}

Die verwendeten Arbeitselektroden wurden aus Gold- bzw. Platin-Drähten angefertigt. Hierzu wurde ein dünner Draht mit einem Durchmesser von $200 \mu \mathrm{m}$ bzw. $25 \mu \mathrm{m}$ in eine Glaskapillare (Mikropipette) eingeführt und die Öffnung durch Epoxidklebstoff verschlossen (Abb. 1). Mit Hilfe eines Skalpells wurde das herausragende Drahtende bündig mit dem Glaskapillarende abgeschnitten. Die durch den Schnitt freigelegte Stirnfläche des Drahts dient als wirksame Fläche, welche beim Einsatz der Elektrode mit dem Lösungsmittel in Berührung kommt. Für den elektrischen Kontakt wird an das andere Ende des dünnen Drahts ein dickerer Kupferdraht angelötet. Durch diese einfache und kostengünstige Herstellungstechnik ist die Fertigung von Elektroden mit wirksamen Flächen im Bereich von einigen Quadratmikrometern möglich. Die geometrische Reduktion der wirksamen Fläche einer Elektrode geht einher mit der Verringerung der Stromstärke bei potentiometrischen Messungen. Dies hat insbesondere für Batteriebetriebene Sensorkonzepte Vorteile. Durch das angegebene Herstellungsverfahren sind prinzipielle Eignungstests, mit Hilfe von CV-Messungen, von "Mikroelektroden“ für elektrochemische Messung in effektiver Weise möglich.

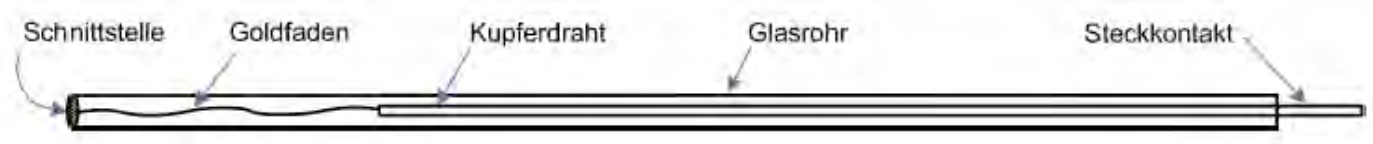

Abb. 1: Schematische Darstestellung des Querschnitts der Arbeitselektrode

Neben dem oben beschriebenen Herstellungsverfahren wurde ein weiteres alternatives Verfahren zur Fertigung von Arbeitselektroden verwendet. Auch hier wurden Au- und Pt-Drähte mit den gleichen Stärken wie zuvor verwendet. Abweichend von obigem Verfahren wurden die Drähte in ein AcrylEinbettmittel eingegossen, wodurch sich die Elektrodenenden mechanisch gut bearbeiten ließen. Die Problematik von undichten Glasröhrchen, welche an den nach dem oben beschriebenen Verfahren hergestellten Elektroden auftreten konnte, wurde damit behoben. Als Gießform wurde eine gläserne Einmalpipette verwendet, die sich nach der ca. 30-minütigen Aushärtung zerbrechen und entfernen ließ. Ein Beispiel für eine auf solche Art gefertigte Elektrode ist in Abb. 2 gezeigt.

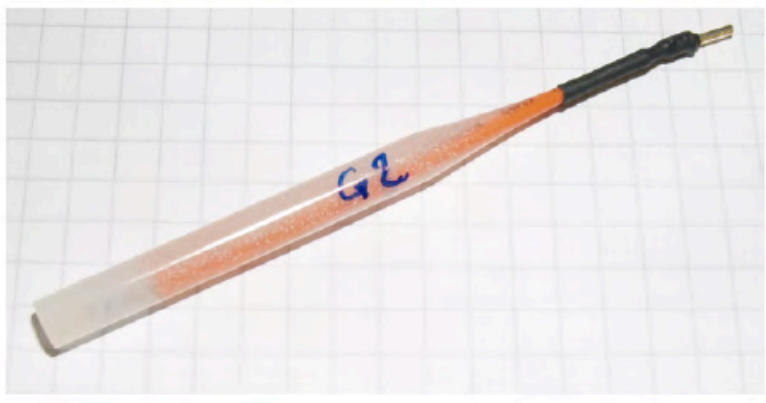

a)

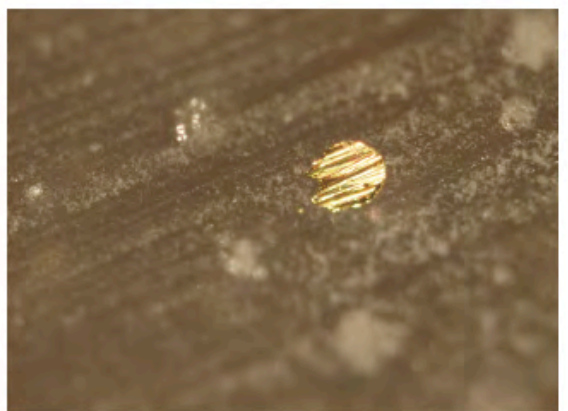

b)

Abb. 2: a)In Arcyl eingebettete Au-Draht-Elektrode. b) Schnittfläche einer Au-Elektrode unter einem Mikroskop

Zusätzlich zu den beschriebenen Drahtelektroden wurden Au-Dünnschicht-Elektroden gefertigt. Dazu wurden vereinzelte goldbeschichtete Waferstücke in den Maßen $15 \mathrm{~mm} \times 15 \mathrm{~mm}$ mit einem verzinnten Kuferdraht verlötet. Damit die Lötstelle nicht in Kontakt mit dem Elektrolyten kommt, wurde sie mit einem Zwei-Komponenten-Epoxykleber hermetisch versiegelt. Die elektroaktive Fläche der Elektrode betrug nach der Versiegelung etwa $90 \mathrm{~mm}^{2}$.

\section{Enzym-Funktionalisierung der Elektroden}

Für die amperometrische Lactatdetektion und voltammetrischen Untersuchungen wurden die Elektroden mit Lactatoxidase funktionalsiert. Die Funktionalsierung der Elektroden wurde jeweils immer kurz vor jeder Messreihe durchgeführt. Generell wurden alle Elektroden vor der Immobilisierung gereinigt, wobei dazu verschiedene Methoden für die Reinigung und deren Einflussnahme auf die Messung untersucht wurden. Jede Elektrode wurde jedoch zumindest mit Ethanol gründlich gereinigt und danach für 15 min 
mit Phosphat-Pufferlösung oder DI-Wasser ins Ultraschall-Bad gelegt. Untersucht wurden zusätzliche Reinigungsschritte wie elektrochemisches Reinigen in Phosphat-Pufferlösung oder Salzsäure $(\mathrm{HCl})$ und Spülen mit Schwefelsäure $\left(\mathrm{H}_{2} \mathrm{SO}_{4}\right)$.

Bei der Immobilisierung von Lactatoxidase mittels chemischer Bindung per Glutaraldehyd wurde die gereinigte Au-Elektrode mit $5 \mu$ l einer Lösung benetzt und für ca. 15 bis 20 min unter einem Abzug getrocknet. Die Zusammensetzung der verwendeten Lösung war wie folgt:

\begin{tabular}{|l|l|}
\hline Substanz & Menge \\
\hline \hline pH-Pufferlösung & $30 \mu \mathrm{l}$ \\
\hline BSA & $10 \mu \mathrm{l}$ \\
\hline Lactatoxidase & $20 \mu \mathrm{l}$ \\
\hline Glutaraldehyd $5 \%$ & $10 \mu \mathrm{l}$ \\
\hline
\end{tabular}

Das Glutaraldehyd wurde stets zuletzt hinzugefügt, da die Quervernetzung innerhalb von 30 bis 60 Sekunden soweit fortgeschritten war, dass eine Benetzung der Elektrodenoberfläche mittels einer Mikroliterpipette nicht mehr möglich war. Die funktionalsierten Elektroden wurden nach der Trocknungszeit entweder direkt verwendet oder in Phosphat-Pufferlösung getaucht aufbewahrt.

\section{Test und Charakterisierung der Elektroden}

Zur Charakterisierung der hergestellten Elektroden wurden zunächst mit nicht-funktionalisierten Arbeitselektroden aus Gold und Platin cyclovoltametrische Messungen in verdünnten $\mathrm{H}_{2} \mathrm{O}_{2}$-Lösungen in Wasser und Phosphatpuffer durchgeführt. Dabei wurde ein Potentialbereich von -800 mV bis $1200 \mathrm{mV}$ (gegen Ag/AgCl-Referenzelektrode) mit einer Scan-Rate von $40 \mathrm{mV} / \mathrm{s}$ durchlaufen. Für die Messungen wurde der Potentiostat $\mu$ AutolabllI der Firma Metrohm GmbH verwendet. Als elektrochemische Zelle wurde VC-3 Voltammetry cell und als Referenzelektrode RE-1B beides von der Firma ALS Co. Ltd, Tokyo

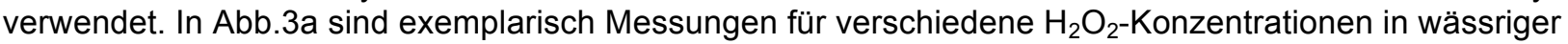
Lösung wiedergegeben. Gezeigt sind Cyclovoltagramme nach jeweils 5 maligem Durchlaufen eines Zykluses. Hiernach waren die nachfolgenden Kurven deckungsgleich mit den vorangegangenen. Die Voltagramme der Messungen von $\mathrm{H}_{2} \mathrm{O}_{2}$ in Reinstwasser (hier nicht gezeigt) weisen keine ausgeprägten Strompeaks auf, die eine Oxidation oder Reduktion des Wasserstoffperoxids aufzeigen. Dennoch besteht zwischen der Stromstärke bei einem bestimmten Potential und der Wasserstoffperoxidkonzentration eine erwartetete Korrelation.

Demgegenüber zeigen die Messungen in Phosphat-Puffer signifikant konzentrationsabhängige Oxidationspeaks (Abb. 3a). Sowohl der höhere Absolutwert der Peakströme als auch die Tatsache, dass die Oxidationspeaks besser ausgeprägt sind, dürfte auf die höhere Leitfähigkeit des Phosphat-Puffers gegenüber Reinstwasser zurückzuführen sein. Die Position des $\mathrm{H}_{2} \mathrm{O}_{2}$-Oxidationspeaks liegt etwa bei $900 \mathrm{mV}$ und bestätigt somit aus der Literatur berichtete Werte ${ }^{2}$. Es besteht eine gute Linearität zwischen den gemessenem Peakströmen und der Wasserstoffperoxidkonzentration mit einer Empfindlichkeit von $31 \mathrm{~mA} / \mathrm{mM}$ (Abb.3b).

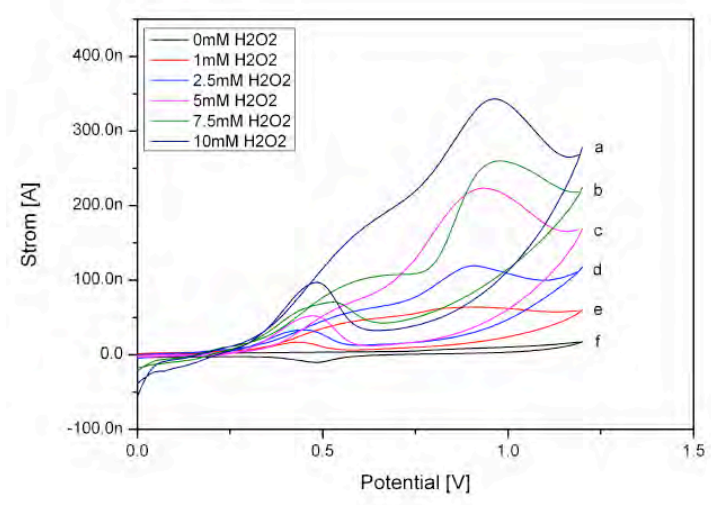

a)

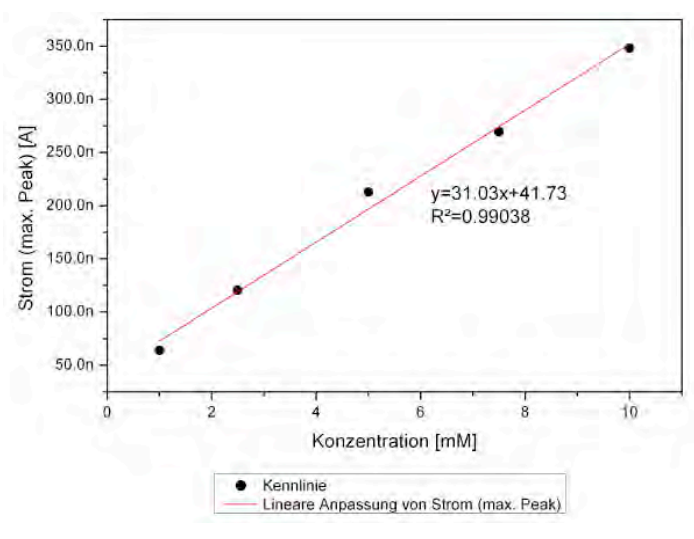

b)

Abb. 3:a) Cyclovoltagramm von $\mathrm{H}_{2} \mathrm{O}_{2}$ in Phosphat-Puffer mit einer $\varnothing 25 \mu \mathrm{m}$-Au-Elektrode bei verschiedenen $\mathrm{H}_{2} \mathrm{O}_{2}$-Konzentrationen. Scan-Rate $=40 \mathrm{mV} / \mathrm{s}$. b) Peak-Stromstärke gegenüber der $\mathrm{H}_{2} \mathrm{O}_{2}$-Konzentration. 
Für die elektrochemische Untersuchung der mit Lactatoxidase funktionalisierten Elektroden wurde eine Reihe von verschieden konzentrierten Lactat-Lösungen angesetzt. Hierzu wurde zunächst eine Basislösung durch Lösen von 45 mg des L-(+)-Lactatsäure-Pulvers in $500 \mathrm{ml}$ Phosphat-Pufferlösung angesetzt und weitere Lösungen wurden durch Verdünnung hergestellt. CV-Messungen an in Arcyl eingebetteten Au-Elektroden weisen deutliche Oxidations- und Reduktionspeaks auf (Abb. 4a). Aus diesen Messungen ergibt sich für Wasserstoffperoxid, das sich über die enzymatische Reaktion mit Lactatoxidase und Lactat-Säure gebildet hat, ein Mittelpunktspotential $E_{m}=720 \mathrm{mV}$. Die Ergebnisse der Oxidations-Peakpotentiale sind mit den in der Literatur vergleichbar ${ }^{2}$. Die Oxidationspeakstromstärke als Funktion der Lactat-Konzentration zeigt ein lineares Verhalten bis zu Lactat-Konzentrationen von $1 \mathrm{mM}$ (Abb. 4b).

Vergleichend zu den Untersuchungen an Drahtelektroden wurden CV-Messungen an funktionalisierten Dünnschicht-Elektroden durchgeführt. Ziel war es zu untersuchen, inwiefern die deutlich größere und dünnere Elektrodenfläche einer gesputterten Goldschicht sich im Vergleich zu einer Au-Drahtelektrode verhält. In Abb.5 sind die CV-Messungen an einer funktionalisierten Au-Dünnschicht-Elektrode gezeigt. Insgesamt ist wie zu erwarten die Stromstärke um fast eine Größenordnung größer als bei den Drahtelektroden, was der größeren Fläche geschuldet ist. Während bei den Messreihen mit den AuDrahtelektroden immer nur ein Redoxpeakpaar sichtbar war, zeigt sich nun ein zweites Peakpaar bei 450 mV bzw. 225 mV (gegen Ag/AgCl-Referenzelektrode). Gerlache et al. ${ }^{2}$ beschreibt ebenfalls ein zweites Redoxpaar im gleichen Potentialbereich, sehen dieses aber für den Einsatz in physiologischen Fluiden nur von begrenztem Nutzen, da darin das amperometrische Signal der Oxidation bei $+450 \mathrm{mV}$ gehemmt wird. Die Kennlinie aus Abb.5b lässt erkennen, dass auch Dünnfilm-Elektroden im Konzentrationsbereich von 0,25 mM bis $1 \mathrm{mM}$ ein lineares Verhalten zeigen. Dabei weist die Elektrode eine Sensitivität von 3,2 $\mathrm{mA} / \mathrm{mM}$ auf.

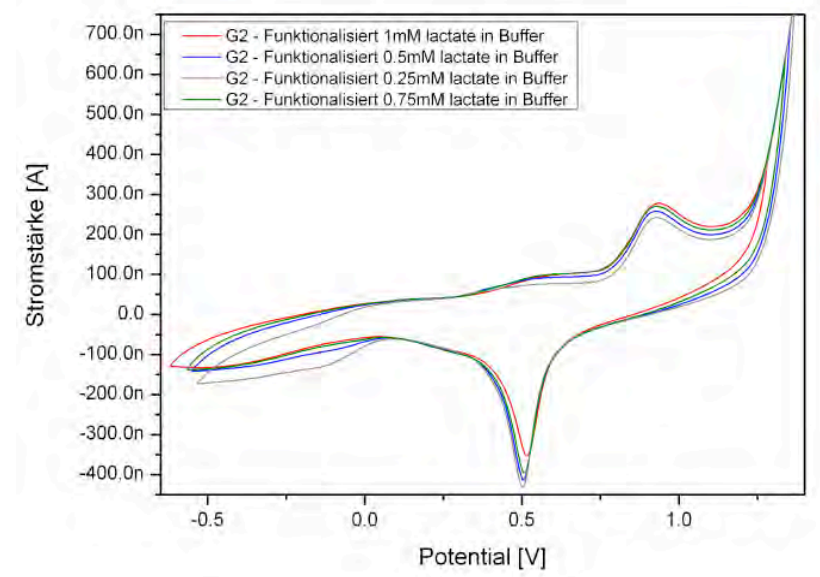

a)

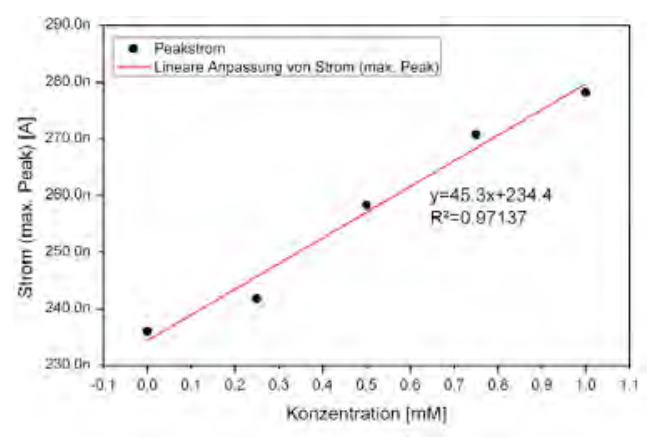

b)

Abb.4: a) CV's mit Ø200 $\mu \mathrm{m}$ Enzym-funktionalisierter Au-Elektrode für verschiedene LactatKonzentrationen. b) Oxidationspeakstromstärke als Funktion der Lactat-Konzentration. 


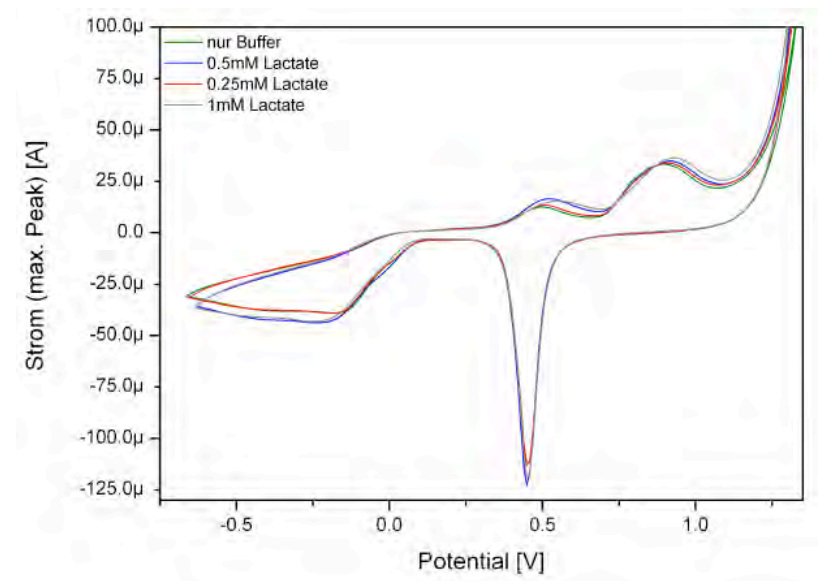

a)

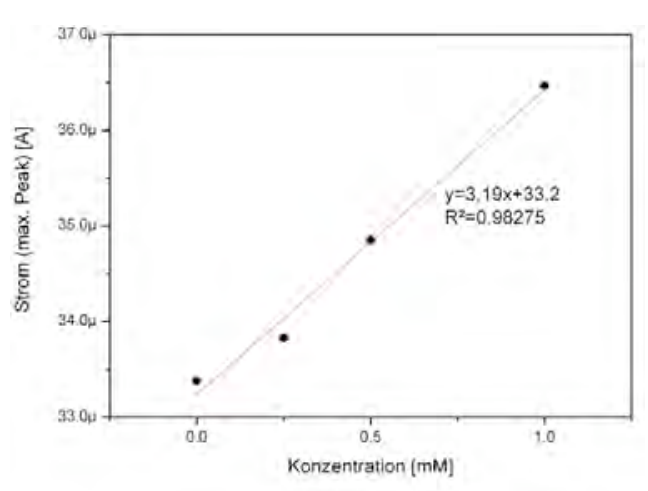

b)

Abb.5: a) CV's mit Au-Dünnschichtelektrode für verschiedene Lactat-Konzentrationen.

b) Oxidationspeakstromstärke des Peaks bei etwa $900 \mathrm{mV}$ als Funktion der Lactat-Konzentration.

Zur Untersuchung der Auswirkungen einer Polymer-Membran auf die Performance wurde Nafion zur Funtionalisierung der Elektrodenoberfläche von Au-Draht-Elektroden verwendet. Romero et al. ${ }^{3}$ und Patel et al. ${ }^{4}$ berichten, dass eine Nafion-Membran die Selektivität der Elektrode erhöht, in dem negativ- bzw. ungeladene Moleküle anderer Spezies den Weg zur Elektrode nicht vollenden können. Nafion ist ein sulfoniertes Tetrafluorethylen-Polymer (PTFE), welches in aliphatischen Alkoholen und Wasser gelöst ist. Ein Zweig in der chemischen Struktur von Nafion ist Sulfonsäure $\left(\mathrm{SO}_{3} \mathrm{H}\right)$. An diesen Zweigen können sich positiv geladene lonen binden und so über Poren durch die Polymer-Matrix transportiert werden. Romero et al. ${ }^{3}$ zeigt ein Verfahren auf, welches zur Herstellung einer flexiblen Membranstruktur mit Hilfe einer Nafion-Lösung verwendet werden kann. Hierbei wurden die Elektroden in eine fertige Nafion-Lösung getaucht. Die leicht flüchtige Lösung hinterlies nach dem Abtrocknen die Polymer-Schicht auf der Elektrodenoberfläche. Durch mehrmaliges Wiederholen konnten so mehrere Schichten von Nafion aufgebracht werden. Die Trocknung fand unter einem Abzug statt und brauchte etwa 5 min pro Schicht. Hiernach erfolgte die Beschichtung mit der Enzym-Matrix. Zur Charakterisierung solch beschichteter Elektroden wurde eine Messreihe mit Enzym-funktionalisierten Au-Drahtelektroden in einer 1-molaren Lactatlösung durchgeführt.

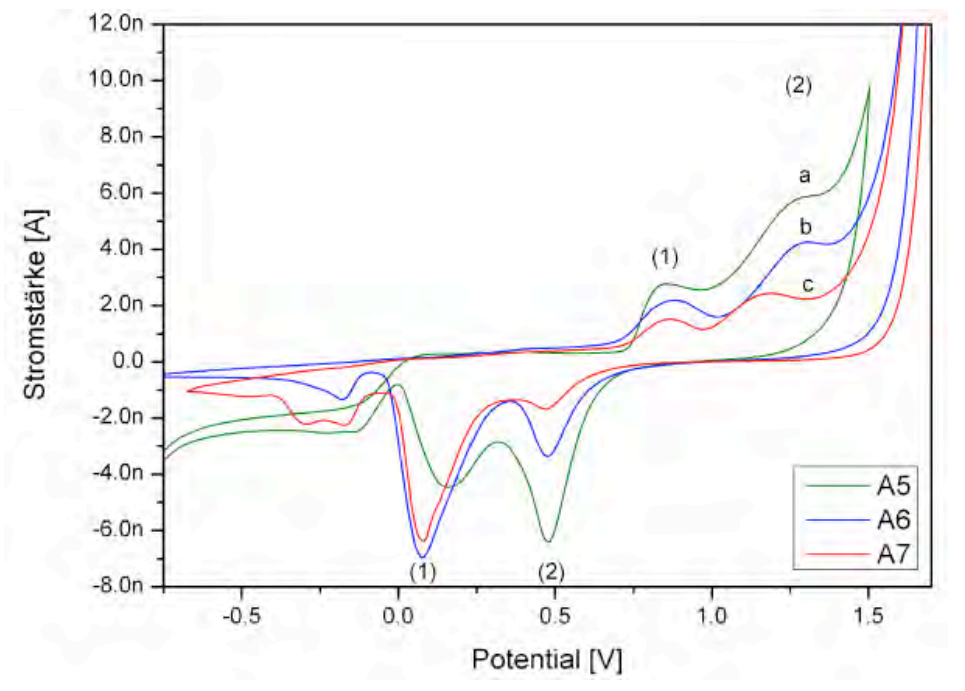

Abb.6: Cyclovoltagramme von $\varnothing 25 \mu \mathrm{m}$-Au-Elektroden mit unterschiedlichen Nafion-Schichtdicken. Schichten auf Elektroden: a) A5 mit einer Schicht, b) A6 mit 2 Schichten, c) A7 mit 3 Schichten.

In den Verläufen der Cyclovoltagramme erkennt man zum einen insgesamt zwei Oxidations- und zwei Reduktionspeaks und zum Anderen den Einfluss der Nafion-Schichtdicke auf den resultierenden Strom. Je dicker die Polymer-Schicht wird, desto geringer der Stromfluss. Desweiteren verschieben sich die Potentiale der insgesamt 4 Peaks leicht. 


\begin{tabular}{|c|c|c|c|c|}
\hline NAFION-SCHICHTEN & $E_{p 1}^{o x}[\mathrm{~V}]$ & $I_{p 1}^{o x}[\mathrm{nA}]$ & $E_{p 1}^{r e d}[\mathrm{~V}]$ & $I_{p 1}^{r e d}[\mathrm{nA}]$ \\
\hline \hline $1 \mathrm{x}$ & 0.860 & 2.77 & 0.156 & -4.46 \\
$2 \mathrm{x}$ & 0.886 & 2.19 & 0.073 & -6.97 \\
$3 \mathrm{x}$ & 0.866 & 1.52 & 0.082 & -6.39 \\
\hline
\end{tabular}

Bei der Betrachtung der Reduktionspeaks fällt auf, dass der Peakstrom $\mathrm{I}_{\mathrm{p} 1}{ }^{\text {red }}$ bei $\mathrm{E}_{\mathrm{p} 1}{ }^{\text {red }}$ mit größerer Schichtdicke betragsmäßig steigt, während $\mathrm{I}_{\mathrm{p} 2}{ }^{\text {red }}$ bei $\mathrm{E}_{\mathrm{p} 2}{ }^{\text {red }}$ kleiner wird (siehe obige Tabelle). Das Auftreten eines zusätzlichen Peakpaares zeigt eine unerwartete Redoxreaktion, welche von einem anderen Stoff verursacht oder von einer Art Verunreinigung hervorgerufen werden könnte. Von einem zweiten Peakpaar wurde auch schon von Gerlache et al. ${ }^{2}$ berichtet. Für die Bestimmung der Konzentration von Wasserstoffperoxid sind die Oxidationspeaks in beiden Potentialbereichen verwendbar, auch wenn sich diese im Vergleich zu den Potentialbereichen ohne Nafion-Membran verschoben haben.

\section{Zusammenfassung}

In der vorliegeneden Arbeit wird die Immobilisierung von Lactatoxidase auf eine Gold-Elektrode mit Hilfe von Glutaraldehyd beschrieben. Da das Enzym auf der einen Seite eine Aktivität weitestgehend behalten und es auf der anderen Seite für eine kontinuierliche Messung fest genug an der Elektrodenoberfläche anhaften soll, mussten geeignete Immobilisierungsmethoden und -rezepturen für einen Lactatsensor entwickelt werden.

Es wurde gezeigt, dass Lactat-Säure mit dem Enzym Lactatoxidase detektierbar ist und dass die Immobilisierungsmethode mit Glutaraldehyd prinzipiell geeignet ist. Desweiteren wurde eine Proportionalität zwischen den gemessenen Peakströmen und der Lactat-Konzentration bis zu Konzentrationen von $1 \mathrm{mM}$ festgestellt.

Die vorgestellte Methodik zur Enzym-Immobilisierung hat deutlich gemacht, dass die richtige Zusammensetzung der Hilfsstoffe und Vernetzungsmittel ebenso ein kritischer Parameter ist, wie die Handhabung der Substanzen und des Enzyms selbst.

\footnotetext{
${ }^{1}$ Iwuoha I. E., Rock A., Smyth R. M., Amperometric L-Lactate Biosensors: 1. Lactic Acid Sensing Electrode Containing Lactate Oxidase in a Composite Poly-L-lysine Matrix, Electroanalysis 11 (1999), No. 5, 367

${ }^{2}$ Gerlache M., Senturk Z., Quarin G., Kaufmann JM, Electrochemical behavior of $\mathrm{H}_{2} \mathrm{O}_{2}$ on gold. Electroanalysis 9 (1997), Nr. 14, 1088-1092.

${ }^{3}$ Romero M.R., Ahumada F., Garay F., Baruzzi A. M., Amperometric Biosensor for Direct Blood Lactate Detection, Analytical Chemistry 82 (2010), 13, 5568-5572

${ }^{4}$ Patel N.G., Erlenkötter A., Cammann K., Chemnitius G.C., Fabrication and characterization of disposable type lactate oxidase for dairy products and clinical analysis., Sensors and Actuators B: Chemical 67 (2000), 1-2, 134-141
} 